\title{
Light Element Production in the Circumstellar Matter of Type Ic Supernovae at Low Metallicity
}

\author{
Ko Nakamura* \\ Department of Astronomy, Graduate School of Science, University of Tokyo \\ Research Center for the Early Universe, Graduate School of Science, University of Tokyo \\ E-mail: nakamura@resceu.s.u-tokyo.ac.jp
}

\section{Susumu Inoue ${ }^{\dagger}$}

\section{Shinya Wanajo}

\section{Toshikazu Shigeyama ${ }^{\S}$}

We investigate energetic Type Ic supernovae as production sites for ${ }^{6} \mathrm{Li}$ and $\mathrm{Be}$ in the early stages of the Milky Way. Recent observations have revealed that some very metal-poor stars with $[\mathrm{Fe} / \mathrm{H}]<-2.5$ possess unexpectedly high abundances of ${ }^{6} \mathrm{Li}$. Some also exhibit enhanced abundances of Be as well as N. From a theoretical point of view, recent studies of the evolution of metal-poor massive stars show that rotation-induced mixing can enrich the outer $\mathrm{H}$ and $\mathrm{He}$ layers with $\mathrm{C}, \mathrm{N}$, and $\mathrm{O}(\mathrm{CNO})$ elements, particularly $\mathrm{N}$, and at the same time cause the intense mass loss of these layers. Here we consider energetic supernova explosions occurring after the progenitor star has lost all but a small fraction of the He layer. The fastest portion of the supernova ejecta and the circumstellar matter (CSM), both of which are composed of He and CNO, can interact directly and induce light-element production through spallation and $\mathrm{He}-\mathrm{He}$ fusion reactions. The CSM should be sufficiently thick to energetic particles so that the interactions terminate within its innermost regions. We calculate the resulting ${ }^{6} \mathrm{Li} / \mathrm{O}$ and ${ }^{9} \mathrm{Be} / \mathrm{O}$ ratios in the ejecta $+\mathrm{CSM}$ material out of which the very metal-poor stars may form. We find that they are consistent with the observed values if the mass of the He layer remaining on the pre-explosion core is $\sim 0.01-0.1 M_{\odot}$ and if the mass fraction of $\mathrm{N}$ mixed in the He layer is $\sim 0.01$. Further observations of ${ }^{6} \mathrm{Li}, \mathrm{Be}$, and $\mathrm{N}$ at low metallicity should provide us with critical tests of this production scenario.

International Symposium on Nuclear Astrophysics - Nuclei in the Cosmos - IX

25-30 June 2006

CERN

\footnotetext{
*Speaker.

$\dagger$ National Astronomical Observatory of Japan

†Department of Astronomy, Graduate School of Science, University of Tokyo

${ }^{\S}$ Research Center for the Early Universe, Graduate School of Science, University of Tokyo
} 


\section{Introduction}

Among the light elements $\mathrm{Li}, \mathrm{Be}$, and $\mathrm{B}(\mathrm{LiBeB}),{ }^{7} \mathrm{Li}$ is thought to arise from a variety of processes, including big bang nucleosynthesis [1] and the $v$-process in Type II supernovae [2]. On the other hand, the main production channel for the rest, in particular for ${ }^{6} \mathrm{Li}$ and $\mathrm{Be}$, is believed to be cosmic-ray-induced nuclear reactions. The most widely discussed models of $\mathrm{LiBeB}$ production are based on cosmic rays accelerated in supernova shocks [3, 4]. Observations of metal-poor stars in the Galactic halo show a primary relation between $[\mathrm{Fe} / \mathrm{H}]$ and $[\mathrm{Be} / \mathrm{H}]$ or $[\mathrm{B} / \mathrm{H}]$, which is consistent with spallation by cosmic rays enriched with $\mathrm{C}, \mathrm{N}$, and $\mathrm{O}(\mathrm{CNO})$ from fresh supernova ejecta impinging on interstellar $\mathrm{H}$ or $\mathrm{He}$ [5].

An alternative possibility was proposed by [6, 7], who considered explosions of Type Ic supernovae ( $\mathrm{SNe} \mathrm{Ic}$ ) as a site for primary $\mathrm{LiBeB}$ production. Since it is expected that a fraction of $\mathrm{C}$ and $\mathrm{O}$ in the surface layers of the ejecta is accelerated to energies above the threshold for spallation reactions when the shock passes through the stellar surface, LiBeB production can occur through the direct interaction of the ejecta with the ambient material, without the need for shock acceleration of cosmic rays. This was explored in greater depth by Nakamura \& Shigeyama (2004)[8], who used more realistic stellar models and equations of state, together with a one-dimensional relativistic hydrodynamic code to investigate in detail how much of the ejecta mass acquires sufficient energies for the LiBeB production. All of these studies used stellar models that completely lost their $\mathrm{H}$ and He envelopes and assumed the target to be interstellar matter (ISM) consisting of $90 \% \mathrm{H}$ and $10 \%$ $\mathrm{He}$, ignoring any circumstellar matter (CSM). Therefore, the only reactions under consideration were $\mathrm{C}, \mathrm{O}+\mathrm{H}, \mathrm{He} \rightarrow \mathrm{LiBeB}$.

Recent observations $[9,10]$ have revealed that some very metal-poor stars possess surprisingly high abundances of ${ }^{6} \mathrm{Li}$, much higher than expected from standard supernova cosmic ray models. The measured values are also higher than can be accommodated in the SN Ic production scenario discussed above, even in the case of an energetic explosion similar to SN 1998bw, as can be seen from the results of Nakamura \& Shigeyama (2004) [8]. However, besides spallation of C and $\mathrm{O}$, the fusion reaction $\mathrm{He}+\mathrm{He} \rightarrow{ }^{6} \mathrm{Li}$ may also be potentially important. This reaction will be significant in the conceivable case that a small fraction of $\mathrm{He}$ is still remaining in the surface layer of the core at the time of the explosion, while most of the He has been transported to the CSM through mass loss. The evolution of metal-poor massive stars has recently been calculated [11, 12] by taking into account the effects of rotation-induced mixing, and it has been shown that extensive mass loss occurs even in extremely metal-poor cases, in addition to significant enrichment of the $\mathrm{N}$ abundance near the surface. If this $\mathrm{N}$ is accelerated at the shock breakout of the $\mathrm{SN}$ explosion, a significant amount of $\mathrm{Be}$ can be produced through the reaction $\mathrm{N}+\mathrm{He} \rightarrow{ }^{9} \mathrm{Be}$, because of its low threshold $E_{\mathrm{th}}\left(\sim 6 \mathrm{MeVA}^{-1}\right)$ and high cross section at peak $\sigma_{\mathrm{p}}(\sim 24$ mbarns) compared with other ${ }^{9} \mathrm{Be}$-producing reactions (e.g., $E_{\mathrm{th}} \sim 8 \mathrm{MeVA}^{-1}$ and $\sigma_{\mathrm{p}} \sim 8 \mathrm{mbarns}$ for $\mathrm{O}+\mathrm{He} \rightarrow{ }^{9} \mathrm{Be}$ ). Indeed, recent observations indicate that the abundances of both $\mathrm{Be}$ and $\mathrm{N}$ may be enhanced in some metal-poor stars in which ${ }^{6} \mathrm{Li}$ is detected $[13,14,15]$. Here, we focus on the interactions $\mathrm{He}, \mathrm{CNO}+\mathrm{He}, \mathrm{N} \rightarrow \mathrm{LiBeB}$ occurring between the ejecta accelerated by the energetic SN explosion and the CSM, in order to account for the abundances in such very metal-poor stars. 


\section{Summary of Observations}

Observational determination of the abundances of ${ }^{6} \mathrm{Li}$ in metal-poor stars is extremely challenging due to its deficiency and to the proximity of its absorption lines to the much stronger ones of the ${ }^{7} \mathrm{Li}$ isotope. Owing to the advent of large telescopes and the improved capabilities of observational instruments, reliable measurements have become possible only in the last several years. 24 metal-poor halo stars were observed [9] with VLT/UVES and ${ }^{6} \mathrm{Li}$ was positively detected in nine of them at the $\geq 2 \sigma$ significance level. The subdwarf LP 815-43 is the most metal-poor object $([\mathrm{Fe} / \mathrm{H}]=-2.74)$ with ${ }^{6} \mathrm{Li}$ in their sample. In the even more metal-poor star G 64-12 $([\mathrm{Fe} / \mathrm{H}]=-3.17)$ a tentative detection with Subaru/HDS was reported [10], although a detailed analysis is still ongoing (W. Aoki et al. 2006, in preparation). With VLT/UVES, the Be abundances of these two stars have been measured $[13,14]$ to be $\log \varepsilon(\mathrm{Be})=-1.09 \pm 0.20$ for LP 815-43 and $-1.10 \pm 0.15$ for G 64-12. The Be abundance in G 64-12 is considerably higher than that expected from previous measurements of the Be-Fe relation in stars with similar metallicities, and this may be the case for LP 815-43 as well. Nitrogen abundances in 31 metal-poor stars were analyzed [15] and it was found that both LP 815-43 and G 64-12 are more N rich than average.

\section{Calculations and Results}

Meynet et al. (2006) [12] and R. Hirschi (2006, in preparation) simulated the evolution of low-metal massive stars with rapid rotation and found that $\mathrm{N}$ is enhanced in the He layers and that they have lost a significant amount of their mass in spite of their initial low metallicities. If there is a considerable amount of $\mathrm{N}$ in the He layers, this $\mathrm{N}$ is accelerated after the shock breakout of $\mathrm{SN}$ explosion and a significant amount of Be will be produced through a reaction $\mathrm{N}+\mathrm{He} \rightarrow$ Be because of its low threshold energy and high cross section at peak. Referring to their results, we consider stellar models which have lost completely their H-rich layers and partly their $\mathrm{He} / \mathrm{N}$ layers as progenitor stars. Here we calculate an explosion of a $\sim 15 M_{\odot}$ star, originated from mainsequence star with mass $\sim 40 M_{\odot}$, with an explosion energy of $3 \times 10^{52}$ ergs corresponding to SN 1998bw [16]. Accelerated ejecta consist of $\mathrm{He}, \mathrm{C}, \mathrm{N}$, and O will collide with circumstellar He and $\mathrm{N}$ stripped from a progenitor star and produce the light elements through spallation reactions and He-He fusion. We use "thick target" approximation to estimate the energy loss of accelerated ejecta assuming that circumstellar He/N is so thick that production of the light elements terminate within the cicumstellar matter and ejecta lose energy mainly by Coulomb collisions with free electrons so that other processes like escaping from a given system can be ignored. Detail is discussed in Nakamura et al. (2006) [17]. The cross sections of the reactions are given by Read \& Viola (1984) [18] and Mercer et al. (2001) [19].

As a result of the localized production of the light elements, their abundance ratios with respect to heavy elements averaged over the CSM and SN ejecta are likely to be inherited by stars of the next generation, as pointed out by Shigeyama \& Tsujimoto (1998) [20]. Thus, we compare the abundance ratios calculated from the above model with the metal-poor stars discussed in the preceding section.

We focus on the abundances of the very metal-poor star LP $815-43$ from which ${ }^{6} \mathrm{Li}, \mathrm{Be}$, and $\mathrm{N}$ have been detected, all at apparently enhanced levels. As seen in the literatures ( $\$ 2)$, 

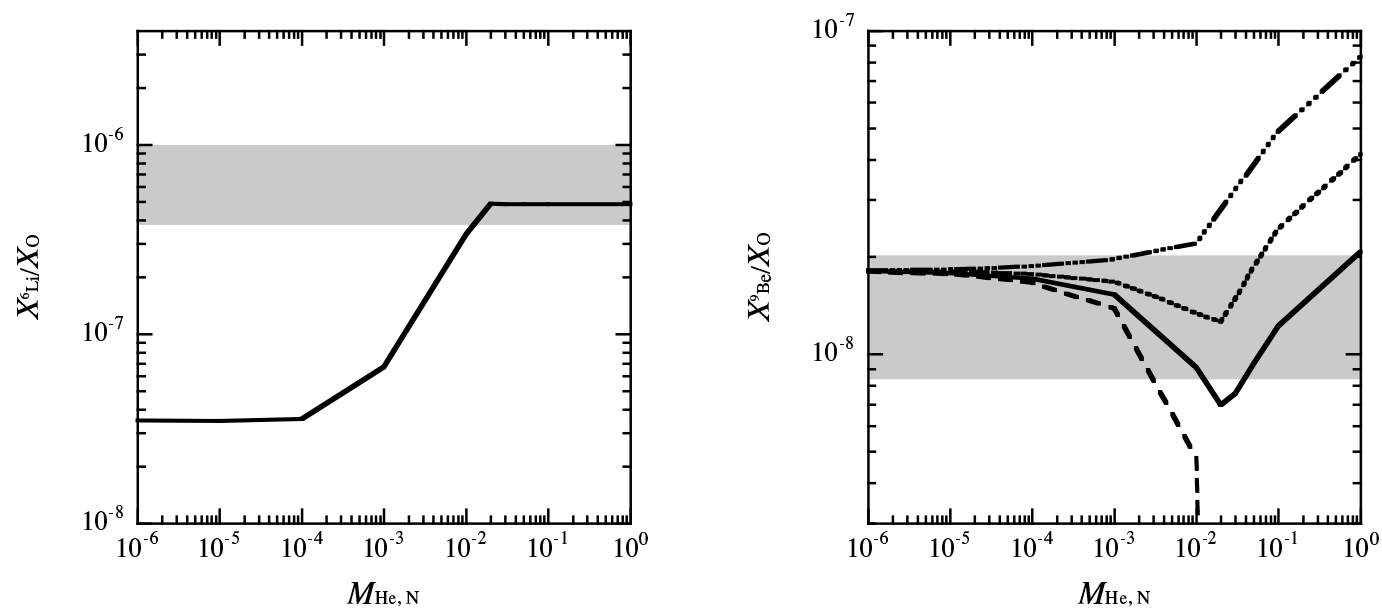

Figure 1: Mass ratios of ${ }^{6} \mathrm{Li}$ (top panel) and ${ }^{9} \mathrm{Be}$ (bottom panel) to $\mathrm{O}$ as functions of $M_{\mathrm{He}, \mathrm{N}}$. Shown are the cases with mass fractions of $\mathrm{N}, X_{\mathrm{N}}=0.005$ (solid line), $X_{\mathrm{N}}=0.01$ (dotted line), $X_{\mathrm{N}}=0.02$ (dashdotted line), and without $\mathrm{N}, X_{\mathrm{N}}=0$ (dashed line). Shaded regions represent the observed ratios for the very metal-poor star LP 815-43 [9, 13], including the error.

$X\left({ }^{6} \mathrm{Li}\right) / X(\mathrm{O}) \sim 6.88_{-3.22}^{+3.08} \times 10^{-7}$ and $X\left({ }^{9} \mathrm{Be}\right) / X(\mathrm{O}) \sim 1.32_{-0.49}^{+0.77} \times 10^{-8}$ for this star. The mass $M_{\mathrm{He}, \mathrm{N}}$ of the $\mathrm{He} / \mathrm{N}$ layer on the preexplosion core and its mass fraction $X_{\mathrm{N}}$ of $\mathrm{N}$ are the main parameters. Figure 1 shows our results. Both $X\left({ }^{6} \mathrm{Li}\right) / X(\mathrm{O})$ and $X\left({ }^{9} \mathrm{Be}\right) / X(\mathrm{O})$ show good agreement with the observational data of LP 815-43 when $M_{\mathrm{He}, \mathrm{N}} \sim 0.01-0.1 M_{\odot}$ and $X_{\mathrm{N}} \sim 0.005-0.01$. These are consistent with recent simulations of the evolution of metal-poor massive stars with rotation ([12]; R. Hirschi 2006, in preparation). The observed $X\left({ }^{9} \mathrm{Be}\right) / X(\mathrm{O})$ for G64-12 can also be reproduced with parameters analogous to those for LP 815-43.

\section{Conclusions and Discussion}

We have proposed a new mechanism to produce the light elements, especially ${ }^{6} \mathrm{Li}$ and ${ }^{9} \mathrm{Be}$ recently observed in metal-poor stars. This is based on recent theoretical findings that rotating, metal-poor massive stars can lose substantial amounts of their envelopes and end up with SNe Ic with a small amount of $\mathrm{He}$ and $\mathrm{N}$ in the outermost layers. The outer layers composed of $\mathrm{He}$ and $\mathrm{CNO}$ are accelerated at the shock breakout and undergo spallation and $\mathrm{He}-\mathrm{He}$ fusion reactions to produce mainly ${ }^{6} \mathrm{Li}$ and ${ }^{9} \mathrm{Be}$. Our calculations show that if $\sim 0.01-0.1 M_{\odot}$ of the He layer is remaining on the core in a SN explosion of a massive star with $M_{\mathrm{ms}}>30 M_{\odot}$, then the observed abundance ratios ${ }^{6} \mathrm{Li} / \mathrm{O}$ can be reproduced. If the He layer contains a small amount $(\sim 0.5 \%-1 \%)$ of $\mathrm{N}$, we can also reproduce ${ }^{9} \mathrm{Be} / \mathrm{O}$.

Compared with the standard picture of cosmic-ray shock acceleration by normal supernovae, our high yield of ${ }^{6} \mathrm{Li}$ results from three crucial differences. First, we consider a large explosion energy, of order $10^{52} \mathrm{ergs}$, as observed in some SNe such as SN 1998bw. Second, our mechanism considers the direct interaction of fast ejecta with the CSM, and it does not involve an efficiency factor for cosmic ray acceleration and is not affected by losses or escape during ISM propagation. Third, the energy distribution of the accelerated particles is a very steeply falling power law, with 
spectral index $\sim 4.6$ as opposed to 2 for shock-accelerated particles. This means that most of the energy is contained in the lowest energy portions, where the cross sections for both $\mathrm{He}+\mathrm{He} \rightarrow{ }^{6} \mathrm{Li}$ and $\mathrm{N}+\mathrm{He} \rightarrow{ }^{9} \mathrm{Be}$ peak.

Both the degree of mass loss and the amount of $\mathrm{N}$ enrichment in the progenitor star are expected to be sensitive to its initial rotation speed $[11,12]$. The actual rotation speed is presumably distributed over a wide range, as is the explosion energy and the extent of mass loss at the time of the explosion. Therefore, dispersions in the ${ }^{6} \mathrm{Li}$ and $\mathrm{Be}$ abundances are expected, and current observations suggest that this may indeed be the case for ${ }^{6} \mathrm{Li}[9,10,21]$ as well as $\mathrm{Be}$ [22]. Our scenario predicts a close relation between $\mathrm{Be}$ and $\mathrm{N}$, since $\mathrm{Be}$ arises directly as a consequence of $\mathrm{N}$ spallation. A looser correlation between ${ }^{6} \mathrm{Li}$ and $\mathrm{N}$ is also expected, as effective ${ }^{6} \mathrm{Li}$ production requires sufficient mass loss, which in turn implies significant $\mathrm{N}$ enrichment. Further observations of ${ }^{6} \mathrm{Li}, \mathrm{Be}$, and $\mathrm{N}$ for a large sample of metal-poor stars should provide us with definitive tests.

We are grateful to Georges Meynet, Raphael Hirschi, Takeru K. Suzuki, and Sean Ryan for valuable discussions. This work has been partially supported by the grants in aid (16540213, 17740108) of the Ministry of Education, Science, Culture, and Sports in Japan.

\section{References}

[1] Spite, F., \& Spite, M. 1982, A \& A, 115, 357

[2] Woosley, S. E., Hartmann, D. H., Hoffman, R. D., \& Haxton, W. C. 1990, ApJ, 356, 272

[3] Meneguzzi, M., Audouze, J. \& Reeves, H. 1971, A \& A, 15, 337

[4] Vangioni-Flam, E., Cassé, M. \& Audouze, J. 2000, Phys. Rep., 333, 365

[5] Duncan, D. K., Lambert, D. L., \& Lemke, M. 1992, ApJ, 401, 584

[6] Fields, B. D., Casse, M., Vangioni-Flam, E., \& Nomoto, K. 1996, ApJ, 462, 276

[7] Fields, B. D., Daigne, F., Cassé, M., \& Vangioni-Flam, E. 2002, ApJ, 581, 389

[8] Nakamura, K. \& Shigeyama, T. 2004, ApJ, 610, 888

[9] Asplund, M., Lambert, D. L., Nissen, P. E., Primas, F., \& Smith, V. V. 2006, ApJ, 644, 229

[10] Inoue, S., Aoki, W., Suzuki, T. K., Kawanomoto, S., García-Pérez, A. E., Ryan, S. G. \& Chiba, M. 2005, From Lithium to Uranium: Elemental Tracers of Early Cosmic Evolution, IAU Symp. 228, ed. V. Hill et al., Cambridge University Press (2005) p.59

[11] Meynet, G., \& Maeder, A. 2002, A \& A, 390, 561

[12] Meynet, G., Ekström, S., \& Maeder, A. 2006, A \& A, 447, 623

[13] Primas, F., Molaro, P., Bonifacio, P., \& Hill, V. 2000a, A \& A, 362, 666

[14] Primas, F., Asplund, M., Nissen, P. E., \& Hill, V. 2000b, A \& A, 364, L42

[15] Israelian, G., Ecuvillon, A., Rebolo, R., García-López, R., Bonifacio, P., \& Molaro, P. 2004, A \& A, 421,649

[16] Nakamura, T., Mazzali, P. A., Nomoto, K., \& Iwamoto, K. 2001, ApJ, 550, 991

[17] Nakamura, K., Inoue, S., Wanajo, S., \& Shigeyama, T. 2006, ApJL, 643, L115

[18] Read, S. M., \& Viola, V. E. 1984, Atomic Data and Nuclear Data Tables, 31, 359 
[19] Mercer, D. J., et al. 2001, Phys. Rev. C, 63, 065805

[20] Shigeyama, T., \& Tsujimoto, T. 1998, ApJL, 507, L135

[21] Aoki, W., Inoue, S., Kawanomoto, S., Ryan, S. G., Smith, I. M., Suzuki, T. K., \& Takada-Hidai, M. 2004, A \& A, 428, 579

[22] Boesgaard, A. M., \& Novicki, M. C. 2006, ApJ, 641, 1122 